Kragujevac Journal of Mathematics

Volume 45(1) (2021), Pages 127-138.

\title{
A NOTE ON PROBABILITY CONVERGENCE DEFINED BY UNBOUNDED MODULUS FUNCTION AND $\alpha \beta$-STATISTICAL CONVERGENCE
}

\author{
SUMIT SOM
}

\begin{abstract}
In this paper we define $f-\alpha \beta$-statistical convergence of order $\gamma$ in probability and $f-\alpha \beta$-strong $p$-Cesàro summability of order $\gamma$ in probability for a sequence of random variables under unbounded modulus function and examine the relation between these two concepts. We show by an example that this notion of $f-\alpha \beta$-statistical convergence of order $\gamma$ in probability is stronger than $\alpha \beta$-statistical convergence of order $\gamma$ in probability [9].
\end{abstract}

\section{INTRODUCTION}

The idea of convergence of a real sequence has been extended to statistical convergence by Fast [10] and Steinhaus [19] and later on reintroduced by Schoenberg [17] independently and is based on the notion of asymptotic density of the subset of natural numbers. However, the first idea of statistical convergence (by different name) was given by Zygmund [20] in the first edition of his monograph published in Warsaw in 1935. Later on it was further investigated from the sequence space point of view and linked with summability theorem by Fridy [11], Connor [5], Šalát [16], Das et. al. [6], Fridy and Orhan [12].

In $[3,4]$ a different direction was given to the study of statistical convergence where the notion of statistical convergence of order $\gamma(0<\gamma<1)$ was introduced by using the notion of natural density of order $\gamma$ (where $n$ is replaced by $n^{\gamma}$ in the denominator in the definition of natural density). It was observed in [3], that the behavior of this new convergence was not exactly parallel to that of statistical convergence and some

Key words and phrases. $\alpha \beta$-statistical convergence, $f$-statistical convergence, $f-\alpha \beta$-statistical convergence of order $\gamma$ in probability, $f-\alpha \beta$-strong $p$-Cesàro summability of order $\gamma$ in probability. 2010 Mathematics Subject Classification. Primary: 40A35. Secondary: 40G15, 60B10.

DOI 10.46793/KgJMat2101.127S

Received: April 08, 2018.

Accepted: October 16, 2018. 
basic properties were obtained. More results on this convergence can be seen from [18].

Recently the idea of statistical convergence of order $\gamma$ was further extended to $\alpha \beta$-statistical convergence of order $\gamma$ in [2] as follows: Let $\alpha=\left\{\alpha_{n}\right\}_{n \in \mathbb{N}}, \beta=\left\{\beta_{n}\right\}_{n \in \mathbb{N}}$ be two non-decreasing sequences of positive real numbers satisfying the conditions, $\alpha_{n} \leq \beta_{n}$ for all $n \in \mathbb{N}$, and $\left(\beta_{n}-\alpha_{n}\right) \rightarrow \infty$ as $n \rightarrow \infty$. This pair of sequence we denoted by $(\alpha, \beta)$. Then a sequence $\left\{x_{n}\right\}_{n \in \mathbb{N}}$ of real numbers is said to be $\alpha \beta$ statistically convergent of order $\gamma$ (where $0<\gamma \leq 1$ ) to a real number $x$ if for each $\varepsilon>0$, the set $K=\left\{n \in \mathbb{N}:\left|x_{n}-x\right| \geq \varepsilon\right\}$ has $\alpha \beta$-natural density zero, i.e.,

$$
\lim _{n \rightarrow \infty} \frac{1}{\left(\beta_{n}-\alpha_{n}+1\right)^{\gamma}}\left|\left\{k \in\left[\alpha_{n}, \beta_{n}\right]:\left|x_{k}-x\right| \geq \varepsilon\right\}\right|=0
$$

and we write $S_{\alpha \beta}^{\gamma}-\lim x_{n}=x$ or $x_{n} \stackrel{S_{\alpha \beta}^{\gamma}}{\longrightarrow} x . \alpha \beta$-statistical convergence of order $\gamma$ is more general than statistical convergence of order $\gamma$, lacunary statistical convergence of order $\gamma$ and $\lambda$ statistical convergence of order $\gamma$ if we take

(i) $\alpha_{n}=1$ and $\beta_{n}=n$, for all $n \in \mathbb{N}$;

(ii) $\alpha_{r}=\left(k_{r-1}+1\right)$ and $\beta_{r}=k_{r}$, for all $r \in \mathbb{N}$, where $\left\{k_{r}\right\}_{r \in \mathbb{N} \cup\{0\}}$ is a lacunary sequence;

(iii) $\alpha_{n}=\left(n-\lambda_{n}+1\right)$ and $\beta_{n}=n$, for all $n \in \mathbb{N}$, respectively.

On the other hand, in probability theory, a new type of convergence called statistical convergence in probability was introduced in [13], as follows: Let $\left\{X_{n}\right\}_{n \in \mathbb{N}}$ be a sequence of random variables where each $X_{n}$ is defined on the same sample space $S$ (for each $n$ ) with respect to a given class of events $\triangle$ and a given probability function $P: \triangle \rightarrow \mathbb{R}$. Then the sequence $\left\{X_{n}\right\}_{n \in \mathbb{N}}$ is said to be statistically convergent in probability to a random variable $X$ (where $X: S \rightarrow \mathbb{R}$ ) if for any $\varepsilon, \delta>0$

$$
\lim _{n \rightarrow \infty} \frac{1}{n}\left|\left\{k \leq n: P\left(\left|X_{k}-X\right| \geq \varepsilon\right) \geq \delta\right\}\right|=0 .
$$

In this case we write $X_{n} \stackrel{P S}{\longrightarrow} X$. The class of all sequences of random variables which are statistically convergent in probability is denoted by $P S$. One can also see $[7,8,14]$ for related works.

In the year 2014, the concept of $f$-statistical convergence was introduced by Aizpuru et al. [1] just by replacing $\left|\left\{k \leq n:\left|x_{k}-c\right| \geq \varepsilon\right\}\right|$ and $\frac{1}{n}$ by $f\left(\left|\left\{k \leq n:\left|x_{k}-c\right| \geq \varepsilon\right\}\right|\right)$ and $\frac{1}{f(n)}$, respectively, where $f$ is an unbounded modulus function. The notion of a modulus function was introduced by Nakano [15]. We recall that a modulus function $f$ is a function from $[0, \infty)$ to $[0, \infty)$ such that

(i) $f(x)=0$ if and only if $x=0$;

(ii) $f(x+y) \leq f(x)+f(y)$ for all $x, y \geq 0$;

(iii) $f$ is increasing and $f$ is continuous from the right at 0 . 
The $f$-density of $K \subset \mathbb{N}$ is denoted by $d_{f}(K)=\lim _{n \rightarrow \infty} \frac{f(|K(n)|)}{f(n)}$. In case of $f$-density, the relation $d_{f}(\mathbb{N} \backslash K)=1-d_{f}(K)$ holds only when $d_{f}(K)=0$. In other all cases the relation can't hold.

In a natural way in this paper we combine the approches of the above mentioned papers and introduce new and more general methods, namely, $f-\alpha \beta$-statistical convergence of order $\gamma$ in probability, $f-\alpha \beta$-strong $p$-Cesàro summability of order $\gamma$ in probability for a sequence of random variables. We mainly investigate their relationship and also make some observations about these classes. In the way we show that the notion of $f-\alpha \beta$-statistical convergence of order $\gamma$ in probability is stronger than $\alpha \beta$-statistical convergence of order $\gamma$ in probability (see [9]). It is important to note that the method of proofs and in particular examples are not analogous to the real case.Throughout the paper $f$ will denote unbounded modulus function.

\section{2. $f-\alpha \beta$-Statistical Convergence of Order $\gamma$ in Probability}

We first introduce the definition of $f-\alpha \beta$-statistical convergence of order $\gamma$ for a sequence of real numbers as follows.

Definition 2.1. Let $\left\{x_{n}\right\}_{n \in \mathbb{N}}$ be a sequence of real numbers and $f$ be an unbounded modulus function. The sequence $\left\{x_{n}\right\}_{n \in \mathbb{N}}$ is said to be $f-\alpha \beta$-statistically convergent of order $\gamma$ to a real number $x$ if for any $\varepsilon>0$

$$
\lim _{n \rightarrow \infty} \frac{1}{f\left(\left(\beta_{n}-\alpha_{n}+1\right)^{\gamma}\right)} f\left(\left|\left\{k \in\left[\alpha_{n}, \beta_{n}\right]:\left|x_{k}-x\right| \geq \varepsilon\right\}\right|\right)=0 .
$$

The class of all real sequences which are $f-\alpha \beta$-statistically convergent of order $\gamma$ is denoted by $S_{\alpha \beta}^{\gamma, f}$.

Now we like to introduce the definition of $f-\alpha \beta$-statistical convergence of order $\gamma$ in probability for a sequence of random variables as follows.

Definition 2.2. Let $(S, \triangle, P)$ be a probability space and $\left\{X_{n}\right\}_{n \in \mathbb{N}}$ be a sequence of random variables where each $X_{n}$ is defined on the same sample space $S$ (for each $n$ ) with respect to a given class of events $\triangle$ and a given probability function $P: \triangle \rightarrow \mathbb{R}$. Then the sequence $\left\{X_{n}\right\}_{n \in \mathbb{N}}$ is said to be $f-\alpha \beta$-statistically convergent of order $\gamma$ (where $0<\gamma \leq 1$ ) in probability to a random variable $X$ (where $X: S \rightarrow \mathbb{R}$ ) if for any $\varepsilon, \delta>0$

$$
\lim _{n \rightarrow \infty} \frac{1}{f\left(\left(\beta_{n}-\alpha_{n}+1\right)^{\gamma}\right)} f\left(\left|\left\{k \in\left[\alpha_{n}, \beta_{n}\right]: P\left(\left|X_{k}-X\right| \geq \varepsilon\right) \geq \delta\right\}\right|\right)=0
$$

or equivalently

$$
\lim _{n \rightarrow \infty} \frac{1}{f\left(\left(\beta_{n}-\alpha_{n}+1\right)^{\gamma}\right)} f\left(\left|\left\{k \in\left[\alpha_{n}, \beta_{n}\right]: 1-P\left(\left|X_{k}-X\right|<\varepsilon\right) \geq \delta\right\}\right|\right)=0 .
$$


In this case we write $S_{\alpha \beta}^{\gamma, f}-\lim P\left(\left|X_{n}-X\right| \geq \varepsilon\right)=0$ or $S_{\alpha \beta}^{\gamma, f}-\lim P\left(\left|X_{n}-X\right|<\varepsilon\right)=1$ or just $X_{n} \stackrel{P S_{\alpha \beta}^{\gamma, f}}{\longrightarrow} X$. The class of all sequences of random variables which are $f-\alpha \beta$ statistically convergent of order $\gamma$ in probability is denoted simply by $P S_{\alpha \beta}^{\gamma, f}$.

In Definition 2.2, if we take $f(x)=x$ then $\left\{X_{n}\right\}_{n \in \mathbb{N}}$ is said to be $\alpha \beta$-statistically convergent of order $\gamma$ in probability to a random variable $X$. So, $f-\alpha \beta$-statistical convergence of order $\gamma$ in probability is a generalization of $\alpha \beta$-statistical convergence of order $\gamma$ in probability for a sequence of random variables.

To show that this is indeed more stronger notion than $\alpha \beta$-statistical convergence of order $\gamma$ in probability, we will now give an example of a sequence of random variables which is $\alpha \beta$-statistically convergent of order $\gamma$ in probability but is not $f-\alpha \beta$-statistically convergent of order $\gamma$ in probability.

Example 2.1. Let a sequence of random variables $\left\{X_{n}\right\}_{n \in \mathbb{N}}$ be defined by $X_{n} \in\left\{\begin{array}{l}\{-1,1\} \text { with probability } \frac{1}{2}, \text { if } n=m^{2} \text { for some } m \in \mathbb{N}, \\ \{0,1\} \text { with probability } P\left(X_{n}=0\right)=\left(1-\frac{1}{n^{2}}\right) \text { and } P\left(X_{n}=1\right)=\frac{1}{n^{2}}, \text { if } n \neq m^{2} \\ \text { for any } m \in \mathbb{N} .\end{array}\right.$

Let $0<\varepsilon, \delta<1$. Then, we have,

$$
P\left(\left|X_{n}-0\right| \geq \varepsilon\right)=1, \quad \text { if } n=m^{2} \text { for some } m \in \mathbb{N},
$$

and

$$
P\left(\left|X_{n}-0\right| \geq \varepsilon\right)=\frac{1}{n^{2}}, \quad \text { if } n \neq m^{2} \text { for any } m \in \mathbb{N} .
$$

Let $\frac{1}{2}<\gamma \leq 1, \alpha_{n}=1, \beta_{n}=n^{2}$, for all $n \in \mathbb{N}$ and $f(x)=\frac{x}{1+x}$ for all $x \geq 0$. Then we have the inequality

$$
\frac{1}{n^{2 \gamma}}\left|\left\{k \in\left[1, n^{2}\right]: P\left(\left|X_{n}-0\right| \geq \varepsilon\right) \geq \delta\right\}\right|=\left(\frac{n}{n^{2 \gamma}}+\frac{d}{n^{2 \gamma}}\right) \rightarrow 0 \text { as } n \rightarrow \infty,
$$

where $d$ is a finite positive integer. So, $X_{n} \stackrel{P S_{\alpha \beta}^{\gamma}}{\longrightarrow} 0$, where $\frac{1}{2}<\gamma \leq 1$. But

$$
\lim _{n \rightarrow \infty} \frac{1}{f\left(\left(\beta_{n}-\alpha_{n}+1\right)^{\gamma}\right)} f\left(\left|\left\{k \in\left[\alpha_{n}, \beta_{n}\right]: P\left(\left|X_{k}-0\right| \geq \varepsilon\right) \geq \delta\right\}\right|\right)=1 .
$$

This shows that $\left\{X_{n}\right\}_{n \in \mathbb{N}}$ is not $f-\alpha \beta$-statistically convergent of order $\gamma$ in probability to 0 .

Theorem 2.1. If a sequence of constants $x_{n} \stackrel{S_{\alpha \beta}^{\gamma, f}}{\longrightarrow} x$, then regarding a constant as a random variable having one point distribution at that point, we may also write $x_{n} \stackrel{P S_{\alpha \beta}^{\gamma, f}}{\longrightarrow} x$.

Proof. Proof is straight forward, so omitted. 
The following example shows that in general the converse of Theorem 2.1 is not true and also shows that there is a sequence $\left\{X_{n}\right\}_{n \in \mathbb{N}}$ of random variables which is $f-\alpha \beta$-statistically convergent in probability to a random variable $\mathrm{X}$ but it is not $f-\alpha \beta$-statistically convergent of order $\gamma$ in probability for $0<\gamma<1$.

Example 2.2. Let $c$ be a rational number between $\gamma_{1}$ and $\gamma_{2}$. Let the probability density function of $X_{n}$ be given by

$$
\begin{gathered}
f_{n}(x)= \begin{cases}1, & \text { where } 0<x<1, \\
0, & \text { otherwise, if } n=\left[m^{\frac{1}{c}}\right] \text { for some } m \in \mathbb{N},\end{cases} \\
f_{n}(x)= \begin{cases}\frac{n x^{n-1}}{2^{n}}, & \text { where } 0<x<2, \\
0, & \text { otherwise, if } n \neq\left[m^{\frac{1}{c}}\right] \text { for any } m \in \mathbb{N} .\end{cases}
\end{gathered}
$$

Now let $0<\varepsilon, \delta<1$. Then

$$
\begin{aligned}
& P\left(\left|X_{n}-2\right| \geq \varepsilon\right)=1, \quad \text { if } n=\left[m^{\frac{1}{c}}\right] \text { for some } m \in \mathbb{N}, \\
& P\left(\left|X_{n}-2\right| \geq \varepsilon\right)=\left(1-\frac{\varepsilon}{2}\right)^{n}, \quad \text { if } n \neq\left[m^{\frac{1}{c}}\right] \text { for any } m \in \mathbb{N} .
\end{aligned}
$$

Now let $\alpha_{n}=1, \beta_{n}=n^{2}, f(x)=\sqrt{x}$ for all $x \geq 0$. Consequently, we have the inequality

$$
\lim _{n \rightarrow \infty} \sqrt{\frac{n^{2 c}-1}{n^{2 \gamma_{1}}}} \leq \lim _{n \rightarrow \infty} \frac{1}{f\left(n^{2 \gamma_{1}}\right)} f\left(\left|\left\{k \in\left[1, n^{2}\right]: P\left(\left|X_{k}-2\right| \geq \varepsilon\right) \geq \delta\right\}\right|\right)
$$

and

$$
\lim _{n \rightarrow \infty} \frac{1}{f\left(n^{2 \gamma_{2}}\right)} f\left(\left|\left\{k \in\left[1, n^{2}\right]: P\left(\left|X_{k}-2\right| \geq \varepsilon\right) \geq \delta\right\}\right|\right) \leq \lim _{n \rightarrow \infty} \sqrt{\frac{n^{2 c}+1}{n^{2 \gamma_{2}}}+\frac{d}{n^{2 \gamma_{2}}}},
$$

where $d$ is a fixed finite positive integer. This shows that $\left\{X_{n}\right\}_{n \in \mathbb{N}}$ is $f-\alpha \beta$-statistically convergent of order $\gamma_{2}$ in probability to 2 but is not $f-\alpha \beta$-statistically convergent of order $\gamma_{1}$ in probability to 2 whenever $\gamma_{1}<\gamma_{2}$ and this is not the usual $f-\alpha \beta$ statistical convergence of order $\gamma$ of real numbers. So, the converse of Theorem 2.1 is not true. Also by taking $\gamma_{2}=1$, we see that $X_{n} \stackrel{P S_{\alpha \beta}^{1, f}}{\longrightarrow} 2$ but $\left\{X_{n}\right\}_{n \in \mathbb{N}}$ is not $f-\alpha \beta$-statistically convergent of order $\gamma$ in probability to 2 for $0<\gamma<1$.

Theorem 2.2 (Elementary properties). (i) If $X_{n} \stackrel{P S_{\alpha \beta}^{\gamma, f}}{\longrightarrow} X$ and $X_{n} \stackrel{P S_{\alpha \beta}^{\gamma, g}}{\longrightarrow} Y$, then $P\{X=Y\}=1$, where $f$ and $g$ are unbounded modulus functions and $0<\gamma \leq 1$.

(ii) If $X_{n} \stackrel{P S_{\alpha \beta}^{\gamma_{1}, f}}{\longrightarrow} X$ and $X_{n} \stackrel{P S_{\alpha \beta}^{\gamma_{2}, f}}{\longrightarrow} Y$, then $P\{X=Y\}=1$ for any $\gamma_{1}, \gamma_{2}$ where $0<\gamma_{1}, \gamma_{2} \leq 1$

(iii) Let $0<\gamma_{1} \leq \gamma_{2} \leq 1$. Then $P S_{\alpha \beta}^{\gamma_{1}, f} \subseteq P S_{\alpha \beta}^{\gamma_{2}, f}$ and this inclusion is strict whenever $\gamma_{1}<\gamma_{2}$. 
Proof. (i) If possible let $P\{X=Y\} \neq 1$. Then, there exists two positive real numbers $\varepsilon$ and $\delta$ such that $P(|X-Y| \geq \varepsilon)=\delta>0$. Then we have

$$
\begin{aligned}
& \lim _{n \rightarrow \infty} \frac{f\left(\beta_{n}-\alpha_{n}+1\right)}{f\left(\left(\beta_{n}-\alpha_{n}+1\right)^{\gamma}\right)} \\
& -\lim _{n \rightarrow \infty} \frac{1}{f\left(\left(\beta_{n}-\alpha_{n}+1\right)^{\gamma}\right)} f\left(\left|\left\{k \in\left[\alpha_{n}, \beta_{n}\right]: P\left(\left|X_{k}-Y\right| \geq \frac{\varepsilon}{2}\right) \geq \frac{\delta}{2}\right\}\right|\right) \\
\leq & \lim _{n \rightarrow \infty} \frac{1}{f\left(\left(\beta_{n}-\alpha_{n}+1\right)^{\gamma}\right)} f\left(\left|\left\{k \in\left[\alpha_{n}, \beta_{n}\right]: P\left(\left|X_{k}-X\right| \geq \frac{\varepsilon}{2}\right) \geq \frac{\delta}{2}\right\}\right|\right),
\end{aligned}
$$

which is impossible because the left hand limit is not 0 whereas the right hand limit is 0 . So, $P\{X=Y\}=1$.

(ii) Proof is straightforward and so is omitted.

(iii) The first part is obvious. The inclusion is proper as can be seen from Example 2.2 .

Remark 2.1. In Theorem 2 [3] it was observed that $m_{0}^{\gamma_{1}} \subset m_{0}^{\gamma_{2}}$ and this inclusion was shown to be strict for at least those $\gamma_{1}, \gamma_{2}$ for which there is a $k \in \mathbb{N}$ such that $\gamma_{1}<\frac{1}{k}<\gamma_{2}$. But Example 2.2 shows that the inequality is strict whenever $\gamma_{1}<\gamma_{2}$.

Corollary 2.1. Let $f$ and $g$ be two unbounded modulus functions and $0<\gamma \leq 1$. Then $P S_{\alpha \beta}^{\gamma, f}=P S_{\alpha \beta}^{\gamma, g}$.

Theorem 2.3. If $X_{n} \stackrel{P S_{\alpha \beta}^{\gamma, f}}{\longrightarrow} X$ (where $f$ is an unbounded modulus function), then $X_{n} \stackrel{P S_{\alpha \beta}^{\gamma}}{\longrightarrow} X$.

Proof. As $X_{n} \stackrel{P S_{\alpha \beta}^{\gamma, f}}{\longrightarrow} X$ so for any $\varepsilon, \delta>0$,

$$
\lim _{n \rightarrow \infty} \frac{1}{f\left(\left(\beta_{n}-\alpha_{n}+1\right)^{\gamma}\right)} f\left(\left|\left\{k \in\left[\alpha_{n}, \beta_{n}\right]: P\left(\left|X_{k}-X\right| \geq \varepsilon\right) \geq \delta\right\}\right|\right)=0 .
$$

Let $\frac{1}{p_{1}}>0$. Then there exists $p \in \mathbb{N}$ such that for all $n \geq p$,

$$
\begin{aligned}
& f\left(\left|\left\{k \in\left[\alpha_{n}, \beta_{n}\right]: P\left(\left|X_{k}-X\right| \geq \varepsilon\right) \geq \delta\right\}\right|\right)<\frac{1}{p_{1}} f\left(\left(\beta_{n}-\alpha_{n}+1\right)^{\gamma}\right) \\
\Rightarrow & f\left(\left|\left\{k \in\left[\alpha_{n}, \beta_{n}\right]: P\left(\left|X_{k}-X\right| \geq \varepsilon\right) \geq \delta\right\}\right|\right) \\
& <\frac{1}{p_{1}} f\left(\frac{\left(\beta_{n}-\alpha_{n}+1\right)^{\gamma}}{p_{1}}+\cdots+\frac{\left(\beta_{n}-\alpha_{n}+1\right)^{\gamma}}{p_{1}}\right) \\
\Rightarrow & f\left(\left|\left\{k \in\left[\alpha_{n}, \beta_{n}\right]: P\left(\left|X_{k}-X\right| \geq \varepsilon\right) \geq \delta\right\}\right|\right) \leq f\left(\frac{\left(\beta_{n}-\alpha_{n}+1\right)^{\gamma}}{p_{1}}\right) \\
\Rightarrow & \left|\left\{k \in\left[\alpha_{n}, \beta_{n}\right]: P\left(\left|X_{k}-X\right| \geq \varepsilon\right) \geq \delta\right\}\right| \leq \frac{\left(\beta_{n}-\alpha_{n}+1\right)^{\gamma}}{p_{1}} \\
\Rightarrow & \lim _{n \rightarrow \infty} \frac{1}{\left(\beta_{n}-\alpha_{n}+1\right)^{\gamma}}\left|\left\{k \in\left[\alpha_{n}, \beta_{n}\right]: P\left(\left|X_{k}-X\right| \geq \varepsilon\right) \geq \delta\right\}\right|=0 .
\end{aligned}
$$


This shows that $X_{n} \stackrel{P S_{\alpha \beta}^{\gamma}}{\longrightarrow} X$.

From Theorem 2.3 and Example 2.1 we see that this notion of $f-\alpha \beta$-statistical convergence of order $\gamma$ in probability is stronger than $\alpha \beta$-statistical convergence of order $\gamma$ in probability (see [9]).

Theorem 2.4. Let $f$ be an unbounded modulus function and $0<\gamma \leq 1$. Let $(\alpha, \beta)$ and $\left(\alpha^{\prime}, \beta^{\prime}\right)$ are two pairs of sequences of positive real numbers such that $\left[\alpha_{n}^{\prime}, \beta_{n}^{\prime}\right] \subseteq\left[\alpha_{n}, \beta_{n}\right]$ for all $n \in \mathbb{N}$ and $f\left(\left(\beta_{n}-\alpha_{n}+1\right)^{\gamma}\right) \leq \varepsilon f\left(\left(\beta_{n}^{\prime}-\alpha_{n}^{\prime}+1\right)^{\gamma}\right)$ for some $\varepsilon>0$. Then we have $P S_{\alpha \beta}^{\gamma, f} \subseteq P S_{\alpha^{\prime} \beta^{\prime}}^{\gamma, f}$.

Proof. Proof is straightforward and so is omitted.

But if the condition of the Theorem 2.4 is violated, then limit may not be unique for two different $(\alpha, \beta)$ 's. We now give an example to show this.

Example 2.3. Let $\alpha=\{(2 n) !\}, \beta=\{(2 n+1) !\}$ and $\alpha^{\prime}=\{(2 n+1) !\}, \beta^{\prime}=\{(2 n+2) !\}$ and $f(x)=\sqrt{x}$ for all $x \geq 0$.

Let us define a sequence of random variables $\left\{X_{n}\right\}_{n \in \mathbb{N}}$ by,

$$
X_{k} \in\left\{\begin{array}{l}
\{-1,1\} \text { with probability } P\left(X_{k}=-1\right)=\frac{1}{k}, P\left(X_{k}=1\right)=\left(1-\frac{1}{k}\right), \\
\text { if }(2 n) !<k<(2 n+1) ! \\
\{-2,2\} \text { with probability } P\left(X_{k}=-2\right)=\frac{1}{k}, P\left(X_{n}=2\right)=\left(1-\frac{1}{k}\right), \\
\text { if }(2 n+1) !<k<(2 n+2) !, \\
\{-3,3\} \text { with probability } P\left(X_{k}=-3\right)=P\left(X_{k}=3\right), \\
\text { if } k=(2 n) ! \text { and } k=(2 n+1) ! .
\end{array}\right.
$$

Let $0<\varepsilon, \delta<1$ and $0<\gamma<1$. Then for the sequence $(\alpha, \beta)$

$$
P\left(\left|X_{k}-1\right| \geq \varepsilon\right)=\frac{1}{k}, \quad \text { if }(2 n) !<k<(2 n+1) !
$$

and

$$
P\left(\left|X_{k}-1\right| \geq \varepsilon\right)=1, \quad \text { if }(2 n+1) !<k<(2 n+2) !
$$

and

$$
P\left(\left|X_{k}-1\right| \geq \varepsilon\right)=1, \quad \text { if } k=(2 n) ! \text { and } k=(2 n+1) !
$$

implies

$\lim _{n \rightarrow \infty} \frac{1}{f\left(((2 n+1) !-(2 n) !+1)^{\gamma}\right)} f\left(\left|\left\{k \in[(2 n) !,(2 n+1) !]: P\left(\left|X_{k}-1\right| \geq \varepsilon\right) \geq \delta\right\}\right|\right)=0$.

So, $X_{n} \stackrel{P S_{\alpha \beta}^{\gamma, f}}{\longrightarrow} 1$.

Similarly, it can be shown that for the sequence $\alpha^{\prime}=\{(2 n+1) !\}$, $\beta^{\prime}=\{(2 n+2) !\}, X_{n} \stackrel{P S_{\alpha^{\prime} \beta^{\prime}}^{\gamma, f}}{\longrightarrow} 2$. 
Definition 2.3. Let $(S, \triangle, P)$ be a probability space and $\left\{X_{n}\right\}_{n \in \mathbb{N}}$ be a sequence of random variables where each $X_{n}$ is defined on the same sample space $S$ (for each $n$ ) with respect to a given class of events $\triangle$ and a given probability function $P: \triangle \rightarrow \mathbb{R}$. A sequence of random variables $\left\{X_{n}\right\}_{n \in \mathbb{N}}$ is said to be $f-\alpha \beta$-strong $p$-Cesàro summable of order $\gamma$ (where $0<\gamma \leq 1$ and $p>0$ is any fixed positive real number) in probability to a random variable $\mathrm{X}$ if for any $\varepsilon>0$,

$$
\lim _{n \rightarrow \infty} \frac{1}{f\left(\left(\beta_{n}-\alpha_{n}+1\right)^{\gamma}\right)} \sum_{k \in\left[\alpha_{n}, \beta_{n}\right]} f\left(\left\{P\left(\left|X_{k}-X\right| \geq \varepsilon\right)\right\}^{p}\right)=0 .
$$

In this case we write $X_{n} \stackrel{P W_{\alpha \beta}^{\gamma, p, f}}{\longrightarrow} X$. The class of all sequences of random variables which are $f-\alpha \beta$-strong $p$-Cesàro summable of order $\gamma$ in probability is denoted simply by $P W_{\alpha \beta}^{\gamma, p, f}$.

Theorem 2.5. Let $f$ be an unbounded modulus function such that $f(x) \leq x$ and $f(a x)=a f(x)$, for all $x \geq 0$ and $a \in \mathbb{R}$. If $X_{n} \stackrel{P W_{\alpha \beta}^{\gamma_{1}, p, f}}{\longrightarrow} X$ and $X_{n} \stackrel{P W_{\alpha \beta}^{\gamma_{2}, p, f}}{\longrightarrow} Y$ (where $p \geq 1)$, then $P\{X=Y\}=1$ for any $\gamma_{1}, \gamma_{2}$ where $0<\gamma_{1}, \gamma_{2} \leq 1$.

Proof. Proof is straight forward, so omitted.

Theorem 2.6. Let $f$ and $g$ be unbounded modulus functions satisfying the conditions stated in Theorem 2.5. If $X_{n} \stackrel{P W_{\alpha \beta}^{\gamma, p, f}}{\longrightarrow} X$ and $X_{n} \stackrel{P W_{\alpha \beta}^{\gamma, p, g}}{\longrightarrow} Y$ (where $p \geq 1$ ), then $P\{X=Y\}=1$.

Proof. If possible let $P\{X=Y\} \neq 1$. Then there exists two positive real numbers $\varepsilon$ and $\delta$ such that $P(|X-Y| \geq \varepsilon)=\delta>0$. Then we have

$$
\begin{aligned}
& \{P(|X-Y| \geq \varepsilon)\}^{p} \leq\left\{P\left(\left|X_{k}-X\right| \geq \frac{\varepsilon}{2}\right)+P\left(\left|X_{k}-Y\right| \geq \frac{\varepsilon}{2}\right)\right\}^{p} \\
\Rightarrow & \sum_{k \in\left[\alpha_{n}, \beta_{n}\right]} f\left((P(|X-Y| \geq \varepsilon))^{p}\right) \leq 2^{p} \sum_{k \in\left[\alpha_{n}, \beta_{n}\right]} f\left(\left(P\left(\left|X_{k}-X\right| \geq \frac{\varepsilon}{2}\right)\right)^{p}\right) \\
& +2^{p} \sum_{k \in\left[\alpha_{n}, \beta_{n}\right]} f\left(\left(P\left(\left|X_{k}-Y\right| \geq \frac{\varepsilon}{2}\right)\right)^{p}\right) \\
\Rightarrow & \frac{f\left(\delta^{p}\right)\left(\beta_{n}-\alpha_{n}+1\right)^{\gamma}}{f\left(\left(\beta_{n}-\alpha_{n}+1\right)^{\gamma}\right)}-2^{p} \frac{1}{f\left(\left(\beta_{n}-\alpha_{n}+1\right)^{\gamma}\right)} \sum_{k \in\left[\alpha_{n}, \beta_{n}\right]} f\left(\left(P\left(\left|X_{k}-Y\right| \geq \frac{\varepsilon}{2}\right)\right)^{p}\right) \\
& \leq 2^{p} \frac{1}{f\left(\left(\beta_{n}-\alpha_{n}+1\right)^{\gamma}\right)} \sum_{k \in\left[\alpha_{n}, \beta_{n}\right]} f\left(\left(P\left(\left|X_{k}-X\right| \geq \frac{\varepsilon}{2}\right)\right)^{p}\right),
\end{aligned}
$$

which is impossible because the left hand limit is not 0 whereas the right hand limit is 0 . So, $P\{X=Y\}=1$.

Corollary 2.2. Let $f$ and $g$ be two unbounded modulus functions satisfying the conditions stated in Theorem 2.5 and $0<\gamma \leq 1, p \geq 1$. Then $P W_{\alpha \beta}^{\gamma, p, f}=P W_{\alpha \beta}^{\gamma, p, g}$. 
Theorem 2.7. $\quad$ (i) Let $0<\gamma_{1} \leq \gamma_{2} \leq 1$. Then $P W_{\alpha \beta}^{\gamma_{1}, p, f} \subseteq P W_{\alpha \beta}^{\gamma_{2}, p, f}$. This inclusion is strict whenever $\gamma_{1}<\gamma_{2}$.

(ii) Let $0<\gamma \leq 1$ and $0<p<q<\infty$. Then $P W_{\alpha \beta}^{\gamma, q, f} \subset P W_{\alpha \beta}^{\gamma, p, f}$.

Proof. (i) The first part of this theorem is straightforward and so is omitted. For the second part we will give an example to show that there is a sequence of random variables $\left\{X_{n}\right\}_{n \in \mathbb{N}}$ which is $f-\alpha \beta$-strong $p$-Cesàro summable of order $\gamma_{2}$ in probability to a random variable $\mathrm{X}$ but is not $f-\alpha \beta$-strong $p$-Cesàro summable of order $\gamma_{1}$ in probability whenever $\gamma_{1}<\gamma_{2}$.

Let $2 c$ be a rational number between $\gamma_{1}$ and $\gamma_{2}$. We consider a sequence of random variables :

$$
X_{n} \in\left\{\begin{array}{l}
\{-1,1\} \text { with probability } \frac{1}{2}, \text { if } n=\left[m^{\frac{1}{c}}\right] \text { for some } m \in \mathbb{N}, \\
\{0,1\} \text { with probability } P\left(X_{n}=0\right)=1-\frac{1}{\sqrt[p]{n^{4}}} \text { and } P\left(X_{n}=1\right)=\frac{1}{\sqrt[p]{n^{4}}}, \\
\text { if } n \neq\left[m^{\frac{1}{c}}\right] \text { for any } m \in \mathbb{N} .
\end{array}\right.
$$

Then we have, for $0<\varepsilon<1$

$$
P\left(\left|X_{n}-0\right| \geq \varepsilon\right)=1, \quad \text { if } n=\left[m^{\frac{1}{c}}\right] \text { for some } m \in \mathbb{N}
$$

and

$$
P\left(\left|X_{n}-0\right| \geq \varepsilon\right)=\frac{1}{\sqrt[p]{n^{4}}}, \quad \text { if } n \neq\left[m^{\frac{1}{c}}\right] \text { for any } m \in \mathbb{N} .
$$

Let $\alpha_{n}=1$ and $\beta_{n}=n^{2}$ and $f(x)=\sqrt{x}$ for all $x \geq 0$. So, we have the inequality

$$
\lim _{n \rightarrow \infty} \frac{n^{2 c}-1}{n^{\gamma_{1}}} \leq \lim _{n \rightarrow \infty} \frac{1}{n^{\gamma_{1}}} \sum_{k \in\left[1, n^{2}\right]} f\left(\left\{P\left(\left|X_{k}-0\right| \geq \varepsilon\right)\right\}^{p}\right)
$$

and

$$
\lim _{n \rightarrow \infty} \frac{1}{n^{\gamma_{2}}} \sum_{k \in\left[1, n^{2}\right]} f\left(\left\{P\left(\left|X_{k}-0\right| \geq \varepsilon\right)\right\}^{p}\right) \leq \lim _{n \rightarrow \infty}\left[\frac{n^{2 c}+1}{n^{\gamma_{2}}}+\frac{1}{n^{\gamma_{2}}}\left(\frac{1}{1^{2}}+\frac{1}{2^{2}}+\cdots+\frac{1}{n^{4}}\right)\right] .
$$

This shows that $X_{n} \stackrel{P W_{\alpha \beta}^{\gamma_{2}, p, f}}{\longrightarrow} 0$ but $\left\{X_{n}\right\}_{n \in \mathbb{N}}$ is not $f-\alpha \beta$-strong $p$-Cesàro summable of order $\gamma_{1}$ in probability to 0 .

(ii) Proof is straightforward and so is omitted.

Theorem 2.8. Let $f$ be an unbounded modulus function such that $f(x) \leq x$ for all $x \geq 0$ and $0<\gamma_{1} \leq \gamma_{2} \leq 1$. Then $P W_{\alpha \beta}^{\gamma_{1}, p, f} \subset P S_{\alpha \beta}^{\gamma_{2}, f}$.

Proof. Let $X_{n} \stackrel{P W_{\alpha \beta}^{\gamma_{1}, p, f}}{\longrightarrow} X$. Then for every $\varepsilon>0$,

$$
\lim _{n \rightarrow \infty} \frac{1}{f\left(\left(\beta_{n}-\alpha_{n}+1\right)^{\gamma_{1}}\right)} \sum_{k \in\left[\alpha_{n}, \beta_{n}\right]} f\left(\left\{P\left(\left|X_{k}-X\right| \geq \varepsilon\right)\right\}^{p}\right)=0 .
$$


Then

$$
\begin{gathered}
\sum_{k \in\left[\alpha_{n}, \beta_{n}\right]} f\left(\left(P\left(\left|X_{k}-X\right| \geq \varepsilon\right)\right)^{p}\right) \geq \sum_{\substack{k \in\left[\alpha_{n}, \beta_{n}\right] \\
P\left(\left|X_{k}-X\right| \geq \varepsilon\right) \geq \delta}} f\left(\left(P\left(\left|X_{k}-X\right| \geq \varepsilon\right)\right)^{p}\right) \\
\Rightarrow \sum_{k \in\left[\alpha_{n}, \beta_{n}\right]} f\left(\left(P\left(\left|X_{k}-X\right| \geq \varepsilon\right)\right)^{p}\right) \geq f\left(\delta^{p}\right) f\left(\left|\left\{k \in\left[\alpha_{n}, \beta_{n}\right]: P\left(\left|X_{k}-X\right| \geq \varepsilon\right) \geq \delta\right\}\right|\right) \\
\Rightarrow \frac{1}{f\left(\left(\beta_{n}-\alpha_{n}+1\right)^{\gamma_{1}}\right)} \sum_{k \in\left[\alpha_{n}, \beta_{n}\right]} f\left(\left(P\left(\left|X_{k}-X\right| \geq \varepsilon\right)\right)^{p}\right) \\
\geq f\left(\delta^{p}\right) \frac{1}{f\left(\left(\beta_{n}-\alpha_{n}+1\right)^{\gamma_{2}}\right)} f\left(\left|\left\{k \in\left[\alpha_{n}, \beta_{n}\right]: P\left(\left|X_{k}-X\right| \geq \varepsilon\right) \geq \delta\right\}\right|\right) .
\end{gathered}
$$

This shows that

$$
\lim _{n \rightarrow \infty} \frac{1}{f\left(\left(\beta_{n}-\alpha_{n}+1\right)^{\gamma_{2}}\right)} f\left(\left|\left\{k \in\left[\alpha_{n}, \beta_{n}\right]: P\left(\left|X_{k}-X\right| \geq \varepsilon\right) \geq \delta\right\}\right|\right)=0 .
$$

This completes the proof.

But the converse of Theorem 2.8 is not generally true as can be seen from the following example.

Example 2.4. Let a sequence of random variables $\left\{X_{n}\right\}_{n \in \mathbb{N}}$ be defined by,

$$
X_{n} \in\left\{\begin{array}{l}
\{-1,1\} \text { with probability } \frac{1}{2}, \text { if } n=m^{m} \text { for some } m \in \mathbb{N}, \\
\{0,1\} \text { with probability } P\left(X_{n}=0\right)=1-\frac{1}{\sqrt[p]{n}}, P\left(X_{n}=1\right)=\frac{1}{\sqrt[p]{n}}, \\
\text { if } n \neq m^{m} \text { for any } m \in \mathbb{N} .
\end{array}\right.
$$

Let $0<\varepsilon<1$ and $f(x)=\sqrt{x}$ for all $x \geq 0$. Then

$$
P\left(\left|X_{n}-0\right| \geq \varepsilon\right)=1, \quad \text { if } n=m^{m} \text { for some } m \in \mathbb{N},
$$

and

$$
P\left(\left|X_{n}-0\right| \geq \varepsilon\right)=\frac{1}{\sqrt[p]{n}}, \quad \text { if } n \neq m^{m} \text { for any } m \in \mathbb{N}
$$

Let $\alpha_{n}=1$ and $\beta_{n}=n^{2}$. It can be easily seen that $X_{n} \stackrel{P S_{\alpha \beta}^{\gamma, f}}{\longrightarrow} 0$ for each $0<\gamma \leq 1$.

Let $H=\left\{n \in \mathbb{N}: n \neq m^{m}\right.$ for any $\left.m \in \mathbb{N}\right\}$. Then

$$
\begin{aligned}
\frac{1}{n^{\gamma}} \sum_{k \in\left[1, n^{2}\right]} f\left(\left\{P\left(\left|X_{k}-0\right| \geq \varepsilon\right)\right\}^{p}\right)= & \frac{1}{n^{\gamma}} \sum_{\substack{k \in\left[1, n^{2}\right] \\
k \in H}} f\left(\left\{P\left(\left|X_{k}-0\right| \geq \varepsilon\right)\right\}^{p}\right) \\
& +\frac{1}{n^{\gamma}} \sum_{\substack{k \in\left[1, n^{2}\right] \\
k \notin H}} f\left(\left\{P\left(\left|X_{k}-0\right| \geq \varepsilon\right)\right\}^{p}\right) \\
& =\frac{1}{n^{\gamma}} \sum_{\substack{k \in\left[1, n^{2}\right] \\
k \in H}} \frac{1}{\sqrt{k}}+\frac{1}{n^{\gamma}} \sum_{\substack{k \in\left[1, n^{2}\right] \\
k \notin H}} 1>\frac{1}{n^{\gamma}} \sum_{k=1}^{n^{2}} \frac{1}{\sqrt{k}}>\frac{1}{n^{\gamma-1}},
\end{aligned}
$$


(since $\sum_{k=1}^{n} \frac{1}{\sqrt{k}}>\sqrt{n}$ for $n \geq 2$ ). So, $X_{n}$ is not $f-\alpha \beta$-strong $p$-Cesàro summable of order $\gamma$ in probability to 0 for $0<\gamma \leq 1$.

\section{ACKNOWLEDGEMENT}

Author would like to thank the respected Referees for his/her careful reading of the paper and for giving several valuable suggestions which has improved the presentation of the paper. The author is also grateful to Prof. Pratulananda Das and Dr. Sanjoy Ghosal for their continuously guidance throughout the Ph.D.

\section{REFERENCES}

[1] A. Aizpuru, M. C. Listan-Garcia and F. Rambla-Barreno, Density by moduli and statistical convergence, Quaest. Math. 37 (2014), 525-530.

[2] H. Aktuglu, Korovkin type approximation theorems proved via $\alpha \beta$-statistical convergence, J. Comput. Appl. Math. 259 (2014), 174-181.

[3] S. Bhunia, P. Das and S. Pal, Restricting statistical convergence, Acta Math. Hungar. 134(1-2) (2012), 153-161.

[4] R. Colak, Statistical convergence of order $\alpha$, Modern Methods in Analysis and its Applications (2010), 121-129.

[5] J. S Connor, The statistical and strong p-Cesaro convergence of sequences, Analysis 8(1-2) (1988), 47-63.

[6] P. Das, S. Ghosal and E. Savas, On generalization of certain summability methods using ideals, Appl. Math. Lett. 24(9) (2011), 1509-1514.

[7] P. Das, S. Ghosal and S. Som, Statistical convergence of order $\alpha$ in probability, Arab. J. Math. Sci. 21(2) (2015), 253-265.

[8] P. Das, S. Ghosal and S. Som, Different types of quasi weighted $\alpha \beta$-statistical convergence in probability, Filomat 31(5) (2017), 1463-1473.

[9] P. Das, S. Som, S. Ghosal and V. Karakaya, A notion of $\alpha \beta$-statistical convergence of order $\gamma$ in probability, Kragujevac J. Math. 42(1) (2018), 51-67.

[10] H. Fast, Sur la convergence statistique, Colloq. Math. 2 (1951), 241-244.

[11] J. A. Fridy, On statistical convergence, Analysis 5 (1985), 301-313.

[12] J. A. Fridy and C. Orhan, Lacunary statistical convergence, Pacific. J. Math. 160 (1993), 43-51.

[13] S. Ghosal, Statistical convergence of a sequence of random variables and limit theorems, Appl. Math. 58(4) (2013), 423-437.

[14] S. Ghosal, Generalized weighted random convergence in probability, Appl. Math. Comput. 249 (2014), 502-509.

[15] H. Nakano, Concave modulars, J. Math. Soc. Japan 5 (1953), 29-49.

[16] T. Šalát, On statistically convergent sequences of real numbers, Math. Slovaca 30 (1980), 139150.

[17] J. I. Schoenberg, The integrability of certain functions and related summability methods, Amer. Math. Monthly 66 (1959), 361-375.

[18] H. Sengul and M. Et, On lacunary statistical convergence of order $\alpha$, Acta Math. Sci. Ser. B (Engl. Ed.) 34(2) (2014), 473-482.

[19] H. Steinhaus, Sur la convergence ordinaire et la convergence asymptotique, Colloq. Math. 2 (1951), 73-74.

[20] A. Zygmund, Trigonometric Series, Cambridge University Press, Cambridge, 1979. 
Research Associate,

Department of Mathematics, School of Science,

Adamas University, JagannathPUR,

Kolkata-700126, West Bengal, India

Email address: somkakdwip@gmail.com 